\title{
ORIENTATIONAL-TUNNELING MODEL OF ONE-DIMENSIONAL MOLECULAR SYSTEMS WITH HYDROGEN BONDS
}

\author{
I. V. Stasyuk, O. L. Ivankiv, N. I. Pavlenko \\ Institute for Condensed Matter Physics of the National Academy of Sciences of Ukraine \\ 1 Svientsitskii Str., UA-290011 Lviv, Ukraine
}

(Received October 4, 1996)

\begin{abstract}
The orientational-tunneling model of linear hydrogen-bonded systems with strong short-range proton correlations is developed in the context of problems of proton dynamics and proton transfer. The proton translational motion on a single bond and the Bjerrum orientational jumps are taken into account. The influence of reorientational motion as well as external electric field on energy spectrum and thermodynamical functions of finite linear H-bonded complexes is investigated. Within the framework of the model proposed the systems with $L$ - and $D$-proton defects are described.

Key words: hydrogen bond, proton transfer.

PACS number(s): 64.60.Cn, 66.30.Lw
\end{abstract}

\section{INTRODUCTION}

Various kinds of physical and biological objects contain the hydrogen-bonded molecular subsystems, in which the hydrogen bonds network has a dominant role in their properties.

In particular, the proton subsystem is especially important for superionic crystals with hydrogen bonds. The structural and ferroelectric phase transitions in these compounds are connected with the rearrangements of hydrogen bonds network, proton ordering and ionic groups reorientation processes. The essential feature of this class of materials is their high-temperature transitions to the so-called superionic (superprotonic) phases, in which the conductivity is accomplished by the fast proton transport. In these phases the specific type of proton disorder is realized when protons are distributed randomly in the sublattice of structurally equivalent positions and the number of positions exceeds the number of protons.

Raman and NMR spectroscopy measurements [1-3] indicate the fast dynamics and reorientations of the neighbouring ionic groups, which accompany the phase transitions and become significantly intensive in superionic phase. These movements together with the drastically increased amplitudes of thermal vibrations (measured by the Debye-Waller factor) assist the proton transport and thereby are responsible to a considerable degree for the appearance of the superprotonic conductivity [4]. Proton interacts with the surroundings dynamics adiabatically, that is the "cold" protons are following a "hot" environment without significant barriers along the diffusion path. This is evident from the experimental investigations $[1,5]$ as well as from the results of the molecular dynamics simulation [6].

Because of this, for the description of the proton transfer process the Grotthuss mechanism is considered generally, which involves the proton movement along the hydrogen bond with the further reorientation caused by "tumbling" (vibrational and librational) of the neighbouring ionic group. In other words the first step of the transport process consists of ionic defect motion and the next one is accompanied by the occurrence of the socalled orientational defect. The existence of such types of defects is also accepted in the models describing the proton transport in ice and the orientational relaxation rates of icy substances. A direct experimental estimate of the orientational (Bjerrum) defect charge was obtained by Hubman [7] as a result of measuring the dielectric constant of ammonia doped ice in comparison with ordinary ice. The energy of Bjerrum defects in ice Ih have been determined in [8] with taking into account the relaxation of the surrounding neighbours at the process of the defect forming.

At the present time there exist many unresolved problems in the theoretical description of the proton transport mechanism. In particular, for superionic (superprotonic) crystals the important question is the considering of proton transfer in random two- and three-dimensional hydrogen-bonded medium. Furthermore, the proton migration due to the ionic group reorientations as well as the motion of proton within the hydrogen bond are caused by the interaction with the phonon bath of surroundings, which results in complicating of the problem. To help resolve these problems, there is a need to pursue the theoretical studies of the proton transport in simpler hydrogen-bonded systems.

As the initial step the chain of hydrogen bonds connecting consecutively ionic groups is considered in this work. The role of such a type of molecular subsystems is of great importance for different processes in physical and biological objects, such as ferroelectrics with hydrogen bonds, ice, liquid water, proteins and others $[9,10]$.

The problem of proton dynamics in such systems is the object of intensive theoretical and experimental study. The ab initio self-consistent field (SCF) calculations performed for the small molecular complexes [10, 11] exhibited the anomalous large proton polarizability, which changes strongly under the influence of the external electric field. In $[12,13]$ a pseudo-spin reduced basis model was proposed for the description of $\mathrm{H}$-bonded molecular systems, taking into account only the proton motion 
along the H-bond. In this paper within the framework of our microscopic approach the orientational degrees of freedom corresponding to rotations of covalent bonds connecting protons with ionic groups are taken into account additionally.

The energy levels, polarization and susceptibility of the proton subsystem with small number of hydrogen bonds are investigated. It is shown that the thermodynamical properties of such molecular systems change strongly with the change of orientational motion frequency and external electric field strength. The systems with $L$ - and $D$-proton defects as well as system with one proton in the hydrogen-bonded chain are described within the model developed.

\section{THE MODEL}

Let us consider the molecular system $A H \cdots C H \cdots C H \cdots B$ containing a chain of $N$ hydrogen bonds between the ions or ionic groups $C$ (Fig. 1); $A$ and $B$ denote ions or ionic groups placed on the ends of the chain.

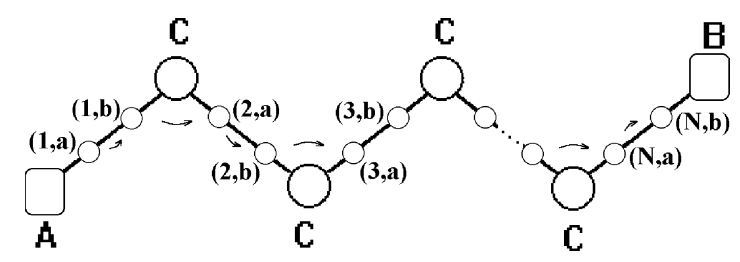

Fig. 1. Scheme of the system. The arrows correspond to one of the possible directions of proton motion along the chain.

For the description of the proton subsystem we shall proceed from the concept implying the availability of a double well potential for the proton on each bond, which is the reason of ionic defect and the existence of orientational (Bjerrum) defect corresponding to the rotation of a covalent bond connecting a proton with ionic group. The ionic defect represents the motion of proton within a single $\mathrm{H}$-bond between the two heavy ions. The orientational defects, on the other hand, allow a proton to move actually along the chain.

In our approximation the two lowest proton states on a bond and only two different orientational positions for the covalent bond are taken into account. We derive the Hamiltonian of the proton subsystem in the second quantization form on the basis of the wave functions $\psi_{a}$ and $\psi_{b}$ of the lowest proton states in the left $(a)$ and right (b) minima on the bond:

$$
\begin{aligned}
& \hat{H}=\hat{H}_{A}+\sum_{k=1}^{N-1} \hat{H}_{k}+\hat{H}_{B}+\hat{H}_{T} \\
& +\hat{H}_{R}+\hat{H}_{C}+\hat{H}_{E}
\end{aligned}
$$

The short-range interaction's part of Hamiltonian includes three terms:

$$
\begin{aligned}
& \hat{H}_{A}=\varepsilon_{A}\left(1-n_{1, a}\right)+w_{A} n_{1, A} \\
& \hat{H}_{k}=w^{\prime}\left(1-n_{k, b}\right)\left(1-n_{k+1, a}\right)+w n_{k, b} n_{k+1, a} \\
& +\varepsilon\left(1-n_{k, b}\right) n_{k+1, a}+\varepsilon n_{k, b}\left(1-n_{k+1, a}\right) \\
& \hat{H}_{B}=\varepsilon_{B}\left(1-n_{N, b}\right)+w_{B} n_{N, b},
\end{aligned}
$$

where $\varepsilon_{A, B}, \varepsilon, w_{A, B}, w, w^{\prime}$ are the energies of proton configurations in the potential minima nearest to the ionic groups (see Fig. 2) and $n_{k, \mu}$ are the occupation numbers of protons in the positions $\mu=a, b$ on the bond $k(k=\overline{1, N})$.

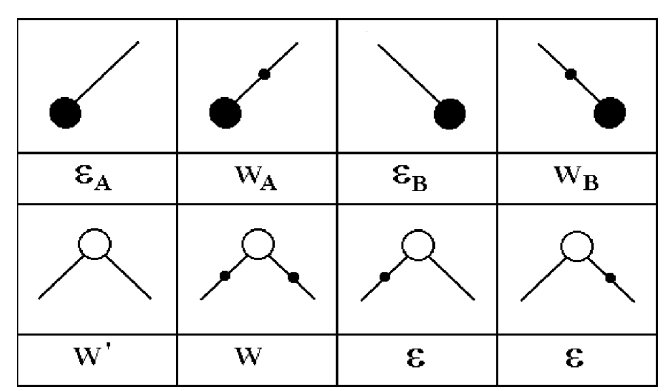

Fig. 2. Proton configurations near ionic groups and their energies.

The tunneling energy $\hat{H}_{T}$ can be presented in the form:

$$
\begin{aligned}
& \hat{H}_{T}=\Omega_{A}\left(c_{1, a}^{\dagger} c_{1, b}+c_{1, b}^{\dagger} c_{1, a}\right) \\
& +\Omega_{B}\left(c_{N, a}^{\dagger} c_{N, b}+c_{N, b}^{\dagger} c_{N, a}\right) \\
& +\Omega_{0} \sum_{k=2}^{N-1}\left(c_{k, a}^{\dagger} c_{k, b}+c_{k, b}^{\dagger} c_{k, a}\right),
\end{aligned}
$$

$c_{k, \mu}^{\dagger}, c_{k, \mu}$ are the proton creation and annihilation operators. We distinguish the tunneling frequencies on the outer $\left(\Omega_{A}, \Omega_{B}\right)$ and inner $\left(\Omega_{0}\right)$ bonds. The effect connected with the orientational motion can be presented as a pseudo-tunneling effect with the frequency $\Omega_{R}$ :

$$
\hat{H}_{R}=\Omega_{R} \sum_{k=1}^{N-1}\left(c_{k, b}^{\dagger} c_{k+1, a}+c_{k+1, a}^{\dagger} c_{k, b}\right) .
$$

The term

$$
\hat{H}_{C}=U \sum_{k=1}^{N} n_{k, a} n_{k, b}+V \sum_{k=1}^{N}\left(1-n_{k, a}\right)\left(1-n_{k, b}\right)
$$




\section{V. STASYUK, O. L. IVANKIV, N. I. PAVLENKO}

corresponds to the energy of the Coulomb repulsion between two protons on the hydrogen bond (the first term) and between free electron pairs, the so called lone pairs $[8,16]$, on the hydrogen bond without protons (the second term). The last term in Hamiltonian (2.1)

$$
\hat{H}_{E}=-e_{p} E \sum_{\substack{k=1 \\ \mu=\{a, b\}}}^{N} R_{k, \mu} n_{k, \mu}
$$

describes the interaction with the external electric field $E ; R_{k, \mu}$ is the distance between the left end of the chain and potential well $(k, \mu) ; e_{p}$ is the proton charge.

In the case of a symmetric system $(A=B)$ some parameters are equal: $\Omega_{A}=\Omega_{B}=\Omega ; \varepsilon_{A}=\varepsilon_{B}=\tilde{\varepsilon}$; $w_{A}=w_{B}=\tilde{w}$.

For the chain with $N$ bonds there exist $2^{2 N}$ manyparticle states $|l\rangle=\left|n_{1, a}, \ldots, n_{N, b}\right\rangle$; where $n_{k, \mu}=\{0,1\}$ is the number of protons in the position $\mu=\{a, b\}$ on the bond $k$. The Hamiltonian expressed in terms of the Hubbard operators $X^{l, l^{\prime}}=|l\rangle\left\langle l^{\prime}\right|$ acting on the basis $|l\rangle$ decomposes into $(2 N+1)$ parts, which correspond to the various values of the proton number $n=\sum_{k, \mu} n_{k, \mu}=0,1, \ldots, 2 N$ :

$$
\hat{H}=\hat{H}^{0} \oplus \hat{H}^{1} \oplus \cdots \oplus \hat{H^{2} N}
$$

The eigenvalues of these Hamiltonians give the energy spectrum of the considered system. The presence of strong short-range correlations between protons on the neighbouring hydrogen bonds as well as a strong Coulomb interaction between protons on a single bond in some finite systems leads to the situation when the possible equilibrium distributions of protons do not contain the high-energy proton configurations. The so called reduced basis model $[12,13]$ is obtained as a result of the exclusion of states corresponding to high-energy shortrange proton configurations. In a similar way the highenergy configurations caused by the Hubbard-type interaction can be excluded also from initial basis.

In particular, for $N=2$, the basis $|l\rangle$ includes 16 states:

$$
\begin{array}{llll}
|1\rangle=|0000\rangle, & |2\rangle=|1000\rangle, & |3\rangle=|0100\rangle, & |4\rangle=|0010\rangle, \\
|5\rangle=|0001\rangle, & |6\rangle=|1100\rangle, & |7\rangle=|1010\rangle, & |8\rangle=|1001\rangle, \\
|9\rangle=|0110\rangle, & |10\rangle=|0101\rangle, & |11\rangle=|0011\rangle, & |12\rangle=|1110\rangle, \\
|13\rangle=|1101\rangle, & |14\rangle=|1011\rangle, & |15\rangle=|0111\rangle, & |16\rangle=|1111\rangle .
\end{array}
$$

With the help of relations

$$
\begin{aligned}
& c_{0, a}=X^{1,2}+X^{3,6}+X^{4,7}+X^{5,8}+X^{9,12}+X^{10,13}+X^{11,14}+X^{15,16} \\
& c_{1, a}=X^{1,4}-X^{2,7}-X^{3,9}+X^{5,11}+X^{6,12}-X^{8,14}-X^{10,15}+X^{13,16} \\
& c_{0, b}=X^{1,3}-X^{2,6}+X^{4,9}+X^{5,10}-X^{7,12}-X^{8,13}+X^{11,15}-X^{14,16} \\
& c_{1, b}=X^{1,5}-X^{2,8}-X^{3,10}-X^{4,11}+X^{6,13}+X^{7,14}+X^{9,15}-X^{12,16},
\end{aligned}
$$

we can express Hamiltonian in terms of $X$ operators. It decomposes into 5 terms:

$$
\hat{H}=\hat{H}_{2}^{0} \oplus \hat{H}_{2}^{1} \oplus \hat{H}_{2}^{2} \oplus \hat{H}_{2}^{3} \oplus \hat{H}_{2}^{4},
$$

where

$$
\begin{aligned}
\hat{H}_{2}^{0}= & a_{2}^{0} \cdot \hat{1}, \\
\hat{H}_{2}^{1}= & \left((b-a)+\left(\mu_{a}+\mu_{a b}\right) E\right) X^{2,2}+\mu_{a} E\left(X^{3,3}-X^{4,4}\right) \\
& +\left((b-a)-\left(\mu_{a}+\mu_{a b}\right) E\right) X^{5,5}+\Omega\left(X^{2,3}+X^{3,2}\right) \\
& +\Omega\left(X^{4,5}+X^{5,4}\right)+\Omega_{R}\left(X^{3,4}+X^{4,3}\right)+a_{2}^{1} \cdot \hat{1}, \\
\hat{H}_{2}^{2}= & \left(U+V+\left(2 \mu_{a}+\mu_{a b}\right) E\right) X^{6,6}+\mu_{a b}\left(X^{7,7}-X^{10,10}\right) \\
& +(b-a) X^{8,8}+(J-(b-a)) X^{9,9}+\left(U+V-\left(2 \mu_{a}+\mu_{a b}\right) E\right) X^{11,11}
\end{aligned}
$$




$$
\begin{aligned}
& +\Omega\left(X^{7,8}+X^{8,7}\right)+\Omega\left(X^{8,10}+X^{10,8}\right)+\Omega\left(X^{9,10}+X^{10,9}\right) \\
& +\Omega_{R}\left(X^{6,7}+X^{7,6}\right)+\Omega_{R}\left(X^{10,11}+X^{11,10}\right)+a_{2}^{2} \cdot \hat{1}, \\
\hat{H}_{2}^{3}= & \left(U+J-(b-a)+\left(\mu_{a}+\mu_{a b}\right) E\right) X^{12,12}+\left(U+\mu_{a} E\right) X^{13,13} \\
& +\left(U-\mu_{a} E\right) X^{14,14}+\left(U+J-(b-a)-\left(\mu_{a}+\mu_{a b}\right) E\right) X^{15,15} \\
& +\Omega\left(X^{12,13}+X^{13,12}\right)+\Omega\left(X^{14,15}+X^{15,14}\right) \\
& +\Omega_{R}\left(X^{13,14}+X^{14,13}\right)+a_{2}^{3} \cdot \hat{1}, \\
\hat{H}_{2}^{4}= & (2 U+J) X^{16,16}+a_{2}^{4} \cdot \hat{1} .
\end{aligned}
$$

Here $(b-a)=(\tilde{w}-\tilde{\varepsilon})-\left(\varepsilon-w^{\prime}\right)$ is the difference between relative energies of proton configurations in the outer (near $A$ or $B$ ionic groups) and inner (near $C$ ) potential wells. The value of $a_{2}^{2}=\tilde{w}+\tilde{\varepsilon}+\varepsilon$ is the energy corresponding to the location of domain walls on the ends of the chain (the domain wall is the boundary between two regions with a varying arrangement of protons on hydrogen bonds);

$$
\begin{aligned}
& a_{2}^{0}=a_{2}^{2}-(\tilde{w}-\tilde{\varepsilon})-\left(\varepsilon-w^{\prime}\right), \\
& a_{2}^{1}=a_{2}^{2}-(\tilde{w}-\tilde{\varepsilon}), \\
& a_{2}^{3}=a_{2}^{2}+(\tilde{w}-\tilde{\varepsilon}), \\
& a_{2}^{4}=a_{2}^{2}+(\tilde{w}-\tilde{\varepsilon})+\left(\varepsilon-w^{\prime}\right) .
\end{aligned}
$$

The parameter $J=w+w^{\prime}-2 \varepsilon$ is the effective shortrange interaction between the protons near ionic group; $\mu_{a}=e_{p} R_{a}, \mu_{a b}=e_{p} R_{a b} ; R_{a}$ and $R_{a b}$ are the distances between the ionic group and the nearest potential well and between the two neighboring potential wells on the hydrogen bond respectively.

In a similar way the Hamiltonian can be written for systems with any finite value of $N$.

\section{ENERGY SPECTRUM AND SUSCEPTIBILITY}

The energy spectrum of the system can be obtained from the equation for eigenvalues and eigenvectors $\left\{u_{l_{\mu}}\right\}$ of Hamiltonian (2.7) represented in the basis $|l\rangle$ :

$$
\sum_{l^{\prime}}\left\langle l|H| l^{\prime}\right\rangle u_{l l^{\prime}}=\lambda_{\mu} u_{l \mu}
$$

The dielectric susceptibility of proton subsystem is expressed by the Green function:

$$
\chi(\omega)=-\frac{2 \pi}{\hbar}\langle\langle P \mid P\rangle\rangle_{\omega} .
$$

Here $P=e_{p} \sum_{k, \mu} R_{k, \mu} n_{k, \mu}$ is the operator of electric dipole moment of protons on the chain. The Hamiltonian represented on the basis $|\tilde{l}\rangle=\sum_{l} u_{l^{\prime}}\left|l^{\prime}\right\rangle$ has the diagonal form:

$$
\tilde{H}=\sum_{p} \lambda_{p} \tilde{X}^{p, p}
$$

where $\tilde{X}^{p, p}$ is obtained by means of a unitary transformation of Hubbard operators:

$$
X^{l, l^{\prime}}=\sum_{\mu \nu} u_{l \mu}^{*} \tilde{X}^{\mu, \nu} u_{\nu l^{\prime}}
$$
$\tilde{X}^{\mu, \nu}$

With the help of the equation of motion for operators

$$
\begin{aligned}
i \hbar \frac{\partial}{\partial t} \tilde{X}^{\mu, \nu}(t) & =\left[\tilde{X}^{\mu, \nu}, \tilde{H}\right]_{t}=\left(\lambda_{\nu}-\lambda_{\mu}\right) \tilde{X}^{\mu, \nu}(t) \\
& =\lambda_{\nu \mu} \tilde{X}^{\mu, \nu}(t),
\end{aligned}
$$

and after substituting (3.4) in (3.2), the susceptibility of the proton subsystem can be written in the form of:

$$
\chi(\omega)=\sum_{\mu<\nu} 2 \tilde{P}_{\mu \nu}^{2} \lambda_{\nu \mu} \frac{\left\langle\tilde{X}^{\mu \mu}-\tilde{X}^{\nu \nu}\right\rangle}{(\hbar \omega)^{2}-\lambda_{\nu \mu}^{2}}
$$

where

$$
\tilde{P}_{\mu \nu}=\sum_{l l^{\prime}} u_{\mu l}\left\langle l|P| l^{\prime}\right\rangle u_{l^{\prime} \nu}^{*}
$$

is the operator of the dipole moment represented on the basis $|\tilde{l}\rangle$. The average occupation number of state $|\mu\rangle$ is determined as:

$$
\left\langle\tilde{X}^{\mu \mu}\right\rangle=\frac{e^{-\beta \lambda_{\mu}}}{\sum_{\nu} e^{-\beta \lambda_{\nu}}} .
$$




\section{ENERGY SPECTRUM AND THERMODYNAMICAL FUNCTIONS FOR SYSTEMS WITH $N=n$}

Let us consider the case of systems in which the number of hydrogen bonds $N$ is equal to the number of protons $n$ in the chain.

In the reduced basis model $[12,13]$, i.e. for systems in which the orientational degrees of freedom can be neglected and after elimination of the high-energy configurations, corresponding to large values of $J$ we have only four parameters of the theory: $(b-a), \Omega, \Omega_{0}, \mu_{a b}$. The values $(b-a)=-600 \mathrm{~cm}^{-1}, \Omega=255 \mathrm{~cm}^{-1}, \Omega_{0}=40 \mathrm{~cm}^{-1}$ were obtained for system formate-water-acid formate from the comparison of the spectrum obtained in the reduced basis model [12] and the SCF calculations [11]. The dipole moment $\mu_{a b}$ is determined from the saturation value of polarization: $\mu_{a b}=3.5 \mathrm{D}$. In a more general case, i.e., taking into account orientational rotations, four additional parameters $U, V, \Omega_{R}$ and $\mu_{a}$ appear. The values of $U=3280 \mathrm{~cm}^{-1}$ and $V=3130 \mathrm{~cm}^{-1}$ correspond to the energies of $D$ - and $L$-defect found in [8]. The parameter $J$ includes the energy of high-energy proton configurations and its value is of one or two order higher than the remaining configuration energies of protons near ionic group (it is in agreement with the results obtained in [11]). Therefore we assume $J=9400 \mathrm{~cm}^{-1} \cdot \mu_{a}=2 D$; $\Omega_{R}$ is changed from 0 to $2500 \mathrm{~cm}^{-1}$.
A. Energy spectrum and susceptibility as a functions of $\Omega_{R}$ for the system with $N=n=2$

For the chain with $n=2$ protons on $N=2$ hydrogen bonds we have 6 basic states:

$$
\begin{aligned}
& |1\rangle=|1100\rangle,|2\rangle=|1010\rangle,|3\rangle=|1001\rangle, \\
& |4\rangle=|0110\rangle,|5\rangle=|0101\rangle,|6\rangle=|0011\rangle .
\end{aligned}
$$

At the absence of tunneling on a hydrogen bond $(\Omega=0)$ the eigenvalues and eigenvectors of Hamiltonian (2.10) were found in the analytical form:

$$
\begin{aligned}
& \lambda_{1,2}=\frac{1}{2}\left(U+V \pm q_{-}\right)+\left(\mu_{a}+\mu_{a b}\right) E+a_{2}^{2}, \\
& \lambda_{3}=(b-a)+a_{2}^{2}, \\
& \lambda_{4}=J-(b-a)+a_{2}^{2}, \\
& \lambda_{5,6}=\frac{1}{2}\left(U+V \pm q_{+}\right)-\left(\mu_{a}+\mu_{a b}\right) E+a_{2}^{2} .
\end{aligned}
$$

here

$$
\begin{aligned}
& u_{1}=\left[\begin{array}{c}
\frac{\Omega_{R}}{\sqrt{\Omega_{R}^{2}+p_{-}^{2}}} \\
-\frac{p_{-}}{\sqrt{\Omega_{R}^{2}+p_{-}^{2}}} \\
0 \\
0 \\
0 \\
0
\end{array}\right] \quad u_{2}=\left[\begin{array}{c}
\frac{p_{-}}{\sqrt{\Omega_{R}^{2}+p_{-}^{2}}} \\
\frac{\Omega_{R}}{\sqrt{\Omega_{R}^{2}+p_{-}^{2}}} \\
0 \\
0 \\
0 \\
0
\end{array}\right] \quad u_{3}=\left[\begin{array}{l}
0 \\
0 \\
1 \\
0 \\
0 \\
0
\end{array}\right] \\
& u_{4}=\left[\begin{array}{l}
0 \\
0 \\
0 \\
1 \\
0 \\
0
\end{array}\right] u_{5}=\left[\begin{array}{c}
0 \\
0 \\
0 \\
0 \\
\frac{\Omega_{R}}{\sqrt{\Omega_{R}^{2}+p_{+}^{2}}} \\
\frac{p_{+}}{\sqrt{\Omega_{R}^{2}+p_{+}^{2}}}
\end{array}\right] u_{6}=\left[\begin{array}{c}
0 \\
0 \\
0 \\
0 \\
-\frac{p_{+}}{\sqrt{\Omega_{R}^{2}+p_{+}^{2}}} \\
\frac{\Omega_{R}}{\sqrt{\Omega_{R}^{2}+p_{+}^{2}}}
\end{array}\right]
\end{aligned}
$$

$$
p_{ \pm}=\frac{1}{2}\left(U+V \pm q_{ \pm}\right) \mp \mu_{a} E, \quad q_{ \pm}=\sqrt{\left(U+V \mp 2 \mu_{a} E\right)^{2}+4 \Omega_{R}^{2}}
$$

The basis $|\tilde{l}\rangle$ has in this case the following form: 


$$
\begin{aligned}
& |\tilde{1}\rangle=\frac{\Omega_{R}}{\sqrt{\Omega_{R}^{2}+p_{-}^{2}}}|1\rangle-\frac{p_{-}}{\sqrt{\Omega_{R}^{2}+p_{-}^{2}}}|2\rangle, \\
& |\tilde{2}\rangle=\frac{p_{-}}{\sqrt{\Omega_{R}^{2}+p_{-}^{2}}}|1\rangle+\frac{\Omega_{R}}{\sqrt{\Omega_{R}^{2}+p_{-}^{2}}}|2\rangle, \\
& |\tilde{3}\rangle=|3\rangle, \\
& |\tilde{4}\rangle=|4\rangle, \\
& |\tilde{5}\rangle=\frac{\Omega_{R}}{\sqrt{\Omega_{R}^{2}+p_{+}^{2}}}|5\rangle+\frac{p_{+}}{\sqrt{\Omega_{R}^{2}+p_{+}^{2}}}|6\rangle, \\
& |\tilde{6}\rangle=-\frac{p_{+}}{\sqrt{\Omega_{R}^{2}+p_{+}^{2}}}|5\rangle+\frac{\Omega_{R}}{\sqrt{\Omega_{R}^{2}+p_{+}^{2}}}|6\rangle .
\end{aligned}
$$

The examination of the spectrum as a function of $\Omega_{R}$ in the absence of external field $E$ shows that the ground state of the proton subsystem is changed at $\Omega_{R}^{*}=\sqrt{(b-a)^{2}-(U+V)(b-a)}$ (see Fig. 3), i.e., the behaviour of the system has two regimes. In the case $\Omega_{R}<\Omega_{R}^{*}$ the ground state $|\tilde{3}\rangle$ corresponds to the location of the domain wall in the centre of the chain, namely when both the protons are in the potential wells near the outer ionic groups $A$ and $B$. For $\Omega_{R}>\Omega_{R}^{*}$ situation is changed; the lowest energy branch $\frac{1}{2}(U+V-q)+a_{2}^{2}$, where $q=\left.q_{+}\right|_{E=0}=\left.q_{-}\right|_{E=0}$, corresponds to the two states $|\tilde{2}\rangle$ and $|\tilde{6}\rangle$ in (4.5). Thus the reconstruction of the ground state reflects the localization of one of the protons near the inner ionic group.

Such a behaviour of the energy spectrum is the reason for the peculiarities in the dependences of thermodynamical functions on pseudotunneling frequency. For example, the free energy of the system for $\Omega=0, E=0$ has the form:

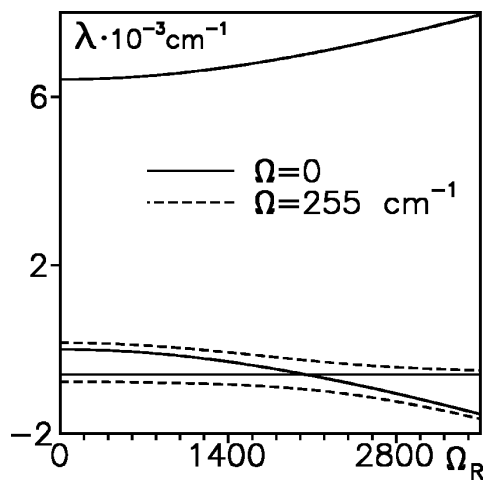

Fig. 3. Dependence of the energy spectrum on $\Omega_{R}$ for the system with $N=n=2$ and different $\Omega$.
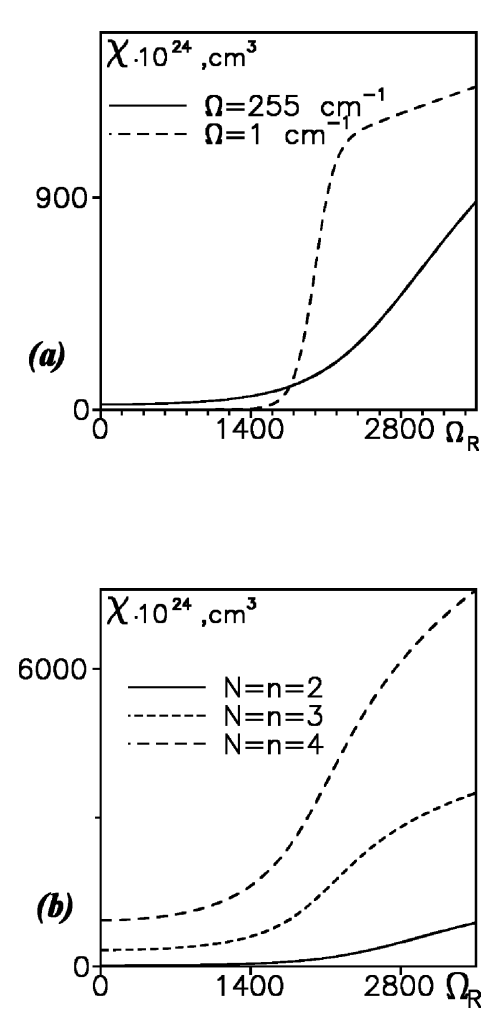

Fig. 4. Static susceptibility as a function of $\Omega_{R}$ at $T=100 K$ a) for the case $\mathrm{N}=\mathrm{n}=2 ;$ b) for different $N$, $\Omega=255 \mathrm{~cm}^{-1}$.

$$
\begin{aligned}
& F=(b-a)-\frac{1}{\beta} \\
& \times \ln \left(1+4 e^{-\frac{\beta}{2}(U+V-2(b-a))} \operatorname{ch} \frac{\beta q}{2}\right) .
\end{aligned}
$$

At very low temperatures $F$ has two distinct branches in dependence on $\Omega_{R}$ :

$$
F^{\beta \rightarrow \infty} \rightarrow \begin{cases}(b-a) & , \quad \Omega_{R}<\Omega_{R}^{*} \\ \frac{1}{2}(U+V-q) & , \Omega_{R}>\Omega_{R}^{*}\end{cases}
$$

With the help of unitary transformations (4.3), (4.4) and using the obtained energy spectrum (4.2), we can calculate $\chi\left(\Omega_{R}\right)$.

For the case of $N=n=2$ at the absence of the external field and for low temperatures the static susceptibility can be represented in the form:

$$
\chi \stackrel{\beta \rightarrow \infty}{\rightarrow} \begin{cases}0 & , \quad \Omega_{R}<\Omega_{R}^{*} \\ 2 \frac{\mu_{a}^{2}}{q}\left(1-\frac{U^{2}}{q^{2}}\right)+\beta\left(\left(\mu_{a}+\mu_{a b}\right)-\frac{U}{q} \mu_{a}\right)^{2} & , \Omega_{R}>\Omega_{R}^{*},\end{cases}
$$

i.e., the anomaly of $\chi\left(\Omega_{R}\right)$ at $\beta \rightarrow \infty$ for $\Omega_{R}>\Omega_{R}^{*}$ (see Fig. 4 , a) reflects the fact, that in the ground state, which is double degenerated, the system possesses a non zero dipole moment. For $\Omega \neq 0$ the energy spectrum and $\chi$ was calculated using numerical methods. The influence of proton tunneling is manifested in the effect of splitting the 
spectrum and smoothing the function $\chi\left(\Omega_{R}\right)$ in the vicinity of $\Omega_{R}=\Omega_{R}^{*}$.

The energy spectrum and susceptibility as a functions of $\Omega_{R}$ was obtained also in a numerical way for the systems with $N=n=3, N=n=4$. We can see, that the change of the ground state of proton subsystems takes place for arbitrary values of $N$. The value of $\Omega_{R}^{*}$ shifts to the low-frequency region with the increase of $N$ (see Fig. 4, b).

\section{B. The dependences of energy spectrum and thermodynamical functions on external electric field}

The dependences of the energy spectrum, average dipole moment $\langle P\rangle$ and susceptibility $\chi$ on the field strength $E[15]$ in the cases of presence or absence of orientational degrees of freedom are different. Neglecting orientational motion we have only a motion of protons between two potential minima on each bond. The redistribution of protons takes place at $E_{1}=\frac{a-b}{\mu_{a b}}$ for the arbitrary values of $N$. Therefore, polarization $\langle P\rangle$ as a function of $E$ abruptly increases at $E=E_{1}$ to the saturated value $\mu_{a b}$. This corresponds to the maximum of susceptibility $\chi$ [12].

In the case when the orientational degrees of freedom are taken into account, the partition function can be calculated with the help of the energy spectrum obtained. As example for case $N=n=2$ :

$$
\begin{aligned}
Z= & e^{-\beta(b-a)}+e^{-\beta(J-(b-a))}+2 e^{-\frac{\beta}{2}(U+V)} \\
& \left(e^{-\beta\left(\mu_{a}+\mu_{a b}\right) E} \operatorname{ch} \frac{\beta q_{-}}{2}+e^{\beta\left(\mu_{a}+\mu_{a b}\right) E} \operatorname{ch} \frac{\beta q_{+}}{2}\right)
\end{aligned}
$$

The average dipole moment of the system can be obtained from $(4.9):\langle P\rangle=-\frac{\partial F}{\partial E}$, where $F=-\frac{1}{\beta} \ln Z$ :

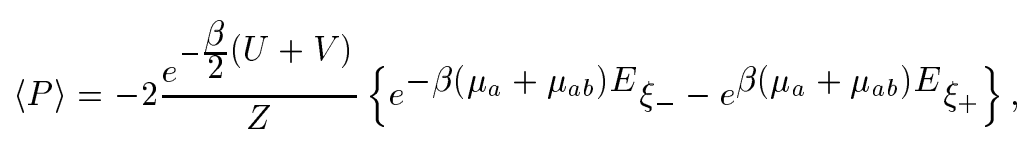

where

$$
\xi_{ \pm}=\left(\mu_{a}+\mu_{a b}\right) \operatorname{ch} \frac{\beta q_{ \pm}}{2}+\mu_{a} \frac{U+V \mp 2 \mu_{a} E}{q_{ \pm}} \operatorname{sh} \frac{\beta q_{ \pm}}{2}
$$

The static susceptibility $\chi(E)$ can be written as:

$$
\begin{aligned}
\chi(E) & =-4 \frac{e^{-\frac{\beta}{2}(U+V)}}{Z^{2}}\left\{e^{\left.-\beta\left(\mu_{a}+\mu_{a b}\right) E_{\xi_{-}}-e^{\beta\left(\mu_{a}+\mu_{a b}\right) E_{\xi_{+}}}\right\}^{2}}\right. \\
& +\frac{e^{-\frac{\beta}{2}(U+V)}}{Z}\left\{e^{\left.-\beta\left(\mu_{a}+\mu_{a b}\right) E_{\eta_{-}}+e^{\beta\left(\mu_{a}+\mu_{a b}\right) E} \eta_{+}\right\}}\right.
\end{aligned}
$$

where

$$
\begin{aligned}
\eta_{ \pm} & =2 \beta\left[\left(\mu_{a}+\mu_{a b}\right)^{2}-\mu_{a}^{2} \frac{\left(U+V \mp 2 \mu_{a} E\right)^{2}}{q_{ \pm}^{2}}\right] \operatorname{ch} \frac{\beta q_{ \pm}}{2} \\
& -4 \frac{\mu_{a}^{2}}{q_{ \pm}}\left[1-\frac{\left(U+V \mp 2 \mu_{a} E\right)^{2}}{q_{ \pm}^{2}}\right] \operatorname{sh} \frac{\beta q_{ \pm}}{2} .
\end{aligned}
$$

Using the obtained expressions (4.2), (4.10) and (4.11) we consider dependences of energy spectrum, $\langle P\rangle$ and $\chi$ on the field strength $E$. The inspection of spectrum for case $\Omega_{R}=0$ in Fig. 5, a shows that the additional reconstruction of the ground state takes place at $E_{2}=\frac{U+V}{2 \mu_{a}}$.
Such a dependence reflects the transfer of all protons in the direction of the applied field to the one end of the chain and, as a result, the appearance of $D$-defect on this end of the chain and $L$-defect on the opposite side. Consequently the additional sharp increasing of average 

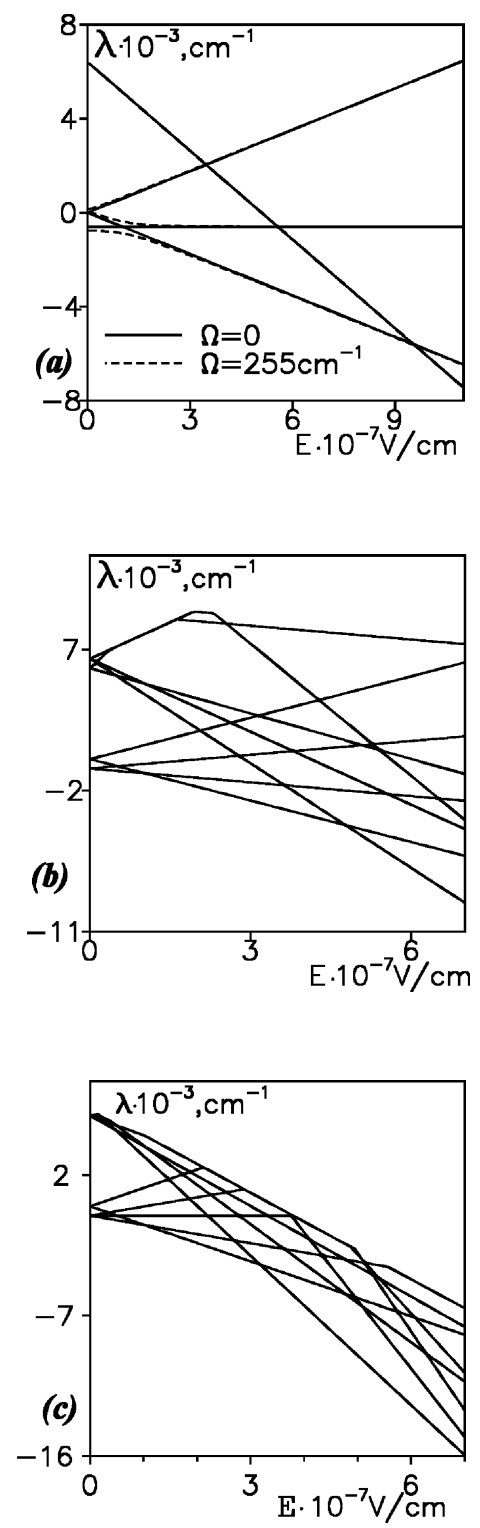

Fig. 5. Dependences of the energy spectrum on $E\left(\Omega_{R}=0\right)$ for the system with a) $N=n=2$, b) $N=n=3$ $\left(\Omega=\Omega_{0}=0\right)$, c) $N=n=4\left(\Omega=\Omega_{0}=0\right)$.

dipole moment $\langle P\rangle$ up to the saturated value $\left(2 \mu_{a}+\mu_{a b}\right)$ takes place at $E=E_{2}$ (Fig. 6, a). Thus we have the anomaly of susceptibility $\chi(E)$ when $E=E_{2}$. We can conclude from Fig. 6 , b, that the first maximum of susceptibility which corresponds to the reconstruction of the spectrum at $E_{1}=\frac{a-b}{\mu_{a b}}$ for $\Omega_{R}=0$, shifts to the lower values of the external electric field strength, when $\Omega_{R}$ increases. The second maximum of $\chi(E)$ at $E=E_{2}$ becomes smoother when $\Omega_{R}$ shifts to the higher-frequency region. Therefore the motion of protons along the chain under the influence of electric field is sensitive to the value of $\Omega_{R}$. When $H$-bonded chain becomes longer, the value of $E_{2}$ changes. For the $N=n=3$ the value of $E_{2}=\frac{U+V}{4 \mu_{a}}$ (Fig. 5, b) and for $N=n=4 E_{2}=\frac{U+V}{6 \mu_{a}}$ (Fig. 5, c). In general $E_{2}=\frac{U+V}{2(N-1) \mu_{a}}$. We see, that for the sufficiently long chains $\left(N>1+\frac{U+V}{a-b} \frac{\mu_{a b}}{2 \mu_{a}}\right)$, the
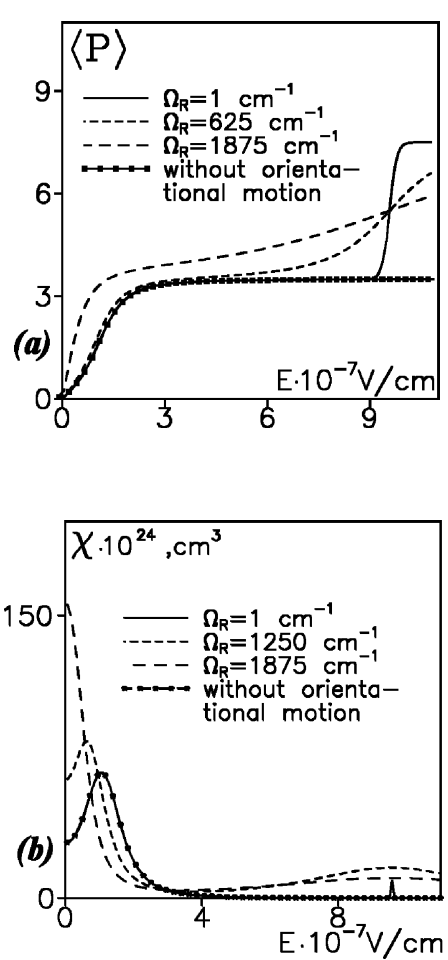

Fig. 6. Dependences of a) the average dipole moment and b) static susceptibility on $E$ for different values of $\Omega_{R}$; the case $N=n=2, T=100 K$.

value of $E_{2}$ is smaller than the value of $E_{1}\left(E_{2}<E_{1}\right)$. Therefore, the appearing of high-energy proton configurations, such as $D$ - and $L$-defects, takes place at the strength of the electric field higher than $E_{2}$, whose value decreases with the increasing of $N$. It is caused by the increasing of a dipole moment of proton subsystem with the rise of the full number of bonds.

\section{V. $L$ - AND $D$-DEFECTS IN THE CHAIN}

In recent decades the research in $\mathrm{H}$-bonded systems has been increasingly focused on the problem of the migration of Bjerrum defects as a possible contribution to various properties of ice, liquid water, ferroelectric crystals with hydrogen bonds and other molecular complexes. The systems with $L$-defects, for which the number of protons or other mobile ions is smaller than the number of possible equilibrium sites are of particular interest. For example, the superionic crystals are the representatives of such a type of structures. It should be noted that such a type of motion is sensitive to the value of $\Omega_{R}$.

\section{A. $L$-defects}

First we consider the case of $n=N-1,(n>1)$, when one $L$-defect takes place in the chain. For the system with $N=3$ hydrogen bonds after neglecting configurations with the energies $\left(V+J-(b-a)+a_{3}^{2}\right)$, $\left(U+V-(b-a)+a_{3}^{2}\right)$, we have 12 basis states: 


$$
\begin{aligned}
|1\rangle & =|110000\rangle,|2\rangle=|101000\rangle,|3\rangle=|100100\rangle, \\
|4\rangle & =|100010\rangle,|5\rangle=|100001\rangle,|6\rangle=|010100\rangle, \\
|7\rangle & =|010010\rangle,|8\rangle=|010001\rangle,|9\rangle=|001010\rangle, \\
|10\rangle & =|001001\rangle,|11\rangle=|000101\rangle,|12\rangle=|000011\rangle .
\end{aligned}
$$

The energy spectrum as a function of $\Omega_{R}$ can be obtained analytically, when $E=0, \Omega=\Omega_{0}=0$ :

$$
\begin{aligned}
& \lambda_{1,2}=\frac{1}{2}(U+3 V \pm q)+a_{3}^{2}, \\
& \lambda_{3,4}=V \pm \Omega_{R}+a_{3}^{2}, \\
& \lambda_{5}=V+(b-a)+a_{3}^{2}, \\
& \lambda_{6,7}=V-(b-a) \pm \sqrt{2} \Omega_{R}+a_{3}^{2}, \\
& \lambda_{8}=V-(b-a)+a_{3}^{2}, \\
& \lambda_{9,10}=V \pm \Omega_{R}+a_{3}^{2}, \\
& \lambda_{11,12}=\frac{1}{2}(U+3 V \pm q)+a_{3}^{2},
\end{aligned}
$$

where $q=\sqrt{(U+V)^{2}+4 \Omega_{R}^{2}}$.

The dependence of energy spectrum (5.2) on $\Omega_{R}$ is shown on Fig. 7 , a. We can observe the reconstructions of the ground state of the system at $\Omega_{R}^{(1)}=-(b-a)$ and $\Omega_{R}^{(2)}=-\frac{b-a}{\sqrt{2}-1}$. For $\Omega_{R}<\Omega_{R}^{(1)}$ the ground state in the basis $|\tilde{l}\rangle$ corresponds to the state $|\tilde{5}\rangle=|5\rangle$ with the energy $\left(V+(b-a)+a_{3}^{2}\right)$, i.e., to the configuration, when both protons are on the different ends of the chain. When $\Omega_{R}^{(1)}<\Omega_{R}<\Omega_{R}^{(2)}$, the ground state of the system is changed, and the proper states are:

$$
\begin{aligned}
|\tilde{4}\rangle & =\frac{1}{\sqrt{2}}(-|3\rangle+|4\rangle), \\
|\tilde{10}\rangle & =\frac{1}{\sqrt{2}}(-|8\rangle+|10\rangle)
\end{aligned}
$$

with the energy $\left(V-\Omega_{R}+a_{3}^{2}\right)$. It corresponds to the localization of one of the protons in a potential well near the inner ionic group in analogy to the case of the absence of $L$-defect in the chain $(N=n)$.

The additional reconstruction of the ground state at $\Omega_{R}=\Omega_{R}^{(2)}$ takes place for the system with $L$-defect and is absent in the case of $N=n$. The ground state

$$
|\tilde{7}\rangle=\frac{1}{\sqrt{2}}|7\rangle-\frac{1}{2}(|6\rangle+|9\rangle)
$$

with the energy $\left(V-(b-a)-\sqrt{2} \Omega_{R}+a_{3}^{2}\right)$ corresponds to the localization of two protons near the inner ionic groups. Such a situation has a small probability when $L$-defect is absent $(N=n)$ because of the high energy of configuration with 2 protons localized near the same ionic group.

The reconstruction of the spectrum leads to the anomalous behaviour of susceptibility. We see the
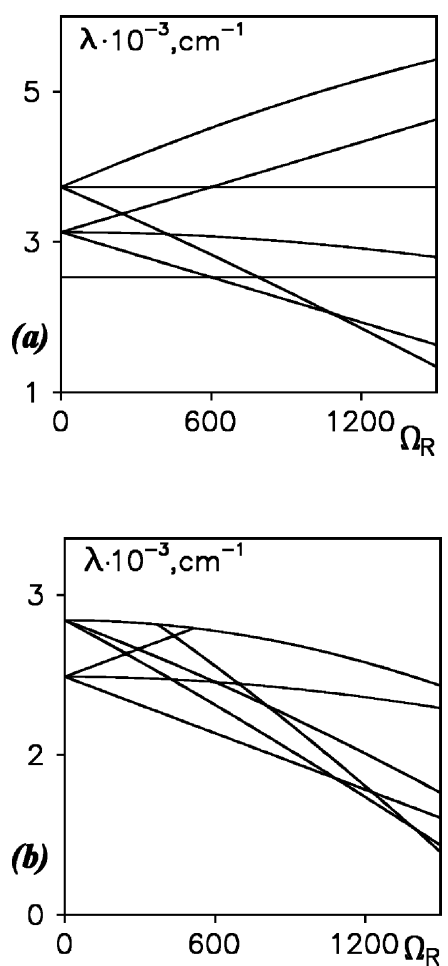

Fig. 7. Dependences of the energy spectrum on $\Omega_{R}$ for the systems with a) $N=3, n=2$, b) $N=4, n=3$; the case of $\Omega=0 \mathrm{~cm}^{-1}, \Omega_{0}=0 \mathrm{~cm}^{-1}$.

decreasing of $\chi\left(\Omega_{R}\right)$ for $\Omega_{R}>\Omega_{R}^{(2)}$ in Fig. 8, a as compared to the case when $L$-defect is absent (see Fig. 4, b).

Such peculiarities take place for the systems with the arbitrary number of hydrogen bonds, when $L$-defect is present in the chain (Fig. 7, b). We can see that the distance between $\Omega_{R}^{(1)}$ and $\Omega_{R}^{(2)}$ becomes smaller with the increase of bonds number (see Fig. 8, b).

The applying of the external electric field leads to the transfer of protons in the direction of the applied field and to the migration of $L$-defect in the opposite direction. We conclude from the dependence of the energy spectrum on $E$, that the ground state of the proton subsystem with the energy $\left(V+(b-a)-(N-3)\left(\mu_{a}+\mu_{a b}\right) E+\right.$ $\left.a_{3}^{2}\right)$ is changed at $E_{1}=\frac{a-b}{2\left(\mu_{a}+\mu_{a b}\right)}$ to the state with the energy value $\left(V+(b-a)-(N-1)\left(\mu_{a}+\mu_{a b}\right) E+a_{3}^{2}\right)$. It corresponds to localization of $L$-defect on the left end of the chain; all the protons are in the right potential wells $(k, b),(k=\overline{2, N})$ on the rest $N-1$ bonds, i.e., they form the ordered subsystem. Thus the left domain wall is placed between the first bond with $L$-defect and second left bond; the right domain wall is on the right end of the chain. The value of $E_{1}=\frac{a-b}{2\left(\mu_{a}+\mu_{a b}\right)}$ remains the same for different $N$. In analogy with the case of $N=n$, the second reconstruction of the spectrum takes place at $E_{2}=\frac{U+V}{2(N-2) \mu_{a}}$. It reflects the appearing of $D$-defect in system on the right end of chain. Configurations with higher energy values appear with a further increasing of $E$. This is the reason for anomalous behaviour of $\langle P\rangle$ and $\chi$ at $E=E_{1}$ and $E=E_{2}$ (Figs.9,a,b). But the dipole 

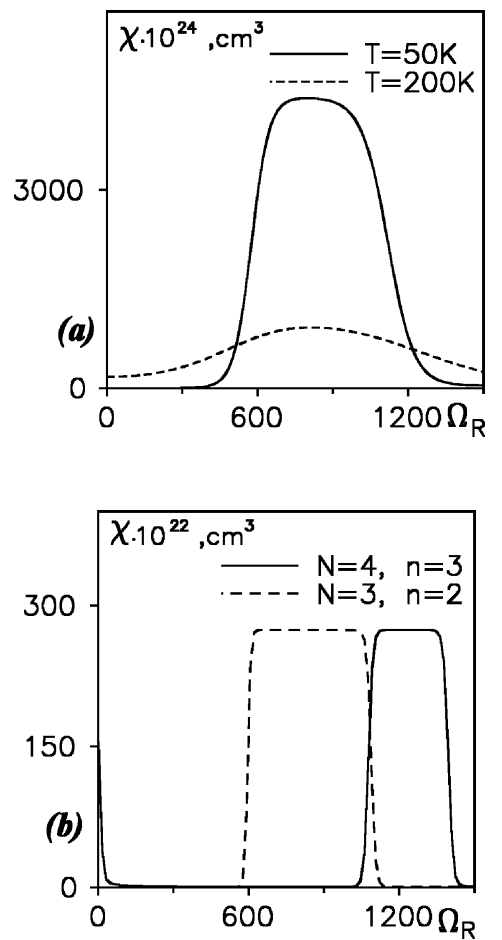

Fig. 8. Dependences of the static susceptibility on $\Omega_{R}$; $\Omega=1 \mathrm{~cm}^{-1}, \Omega_{0}=1 \mathrm{~cm}^{-1}$ a) for the system with $N=4$, $n=3, \mathrm{~b})$ for different $N$ at $T=10 K$.
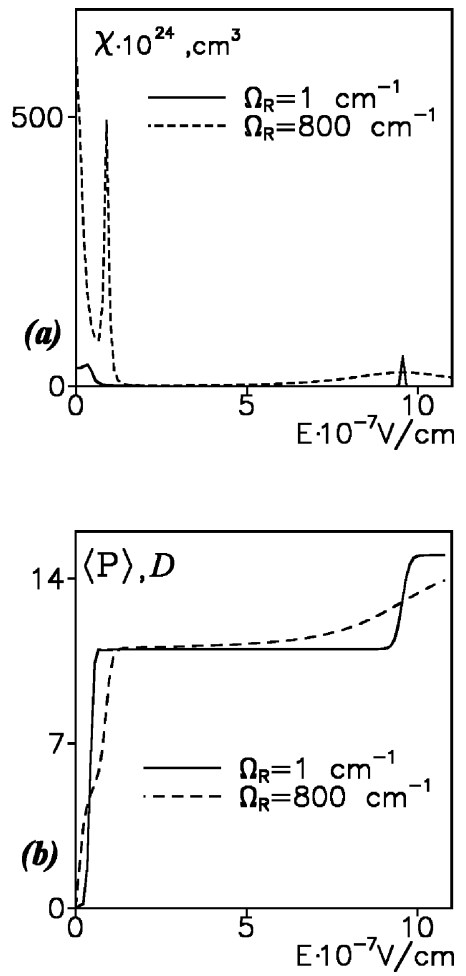

Fig. 9. Dependences of a) the static susceptibility and b) polarization on $E$ for the system with $N=3, n=2$, $\Omega=255 \mathrm{~cm}^{-1}, \Omega_{0}=40 \mathrm{~cm}^{-1}, T=100 \mathrm{~K}$.

moment of the proton subsystem increases so much with the growth of $N$, that for $N>2+\frac{U+V}{a-b}\left(1+\frac{\mu_{a b}}{\mu_{a}}\right)$ we have $E_{2}<E_{1}$, i.e., the high-energy proton configurations appear without the preliminary ordering of the proton subsystem. It should be noted, that it is similar to the case of $N=n$; only the value of the electric field strength, for which the reconstruction of ground state takes place, is different.

When $\Omega_{R}$ increases, one or two protons (depending on the value of $\Omega_{R}$ ) are localized in the inner potential wells of the chain. Thus the application of the electric field leads to the shift of the inner protons in the direction of the applied field to the end of the chain (it corresponds to the first abrupt increase of $\langle P\rangle$ in Fig. 9, $\mathrm{b}$ and the first maximum of $\chi(E)$ in Fig .9, a) and to the grouping of protons on the right end of the chain with a further increase of $E$ (the second shoulder of $\langle P\rangle$ and the maximum of $\chi(E))$. We see, that $\chi(E)$ in the vicinity of $E=E_{2}$ becomes smoother and lower with increasing $\Omega_{R}$, so the behaviour of $\chi(E)$ and $\langle P(E)\rangle$ at $E>E_{1}$ and for different $\Omega_{R}$ is similar to the case of $N=n$.

\section{B. $D$-defects}

For the system with one excess proton in the chain ( $D$-defect) in the case of $E=0$ and $N=2$ the energy spectrum can be written as:

$$
\begin{aligned}
& \lambda_{1,2}=\frac{1}{2}\left(J-(b-a)+2 U+\Omega_{R} \pm q_{-}\right)+a_{2}^{3}, \\
& \lambda_{3,4}=\frac{1}{2}\left(J-(b-a)+2 U-\Omega_{R} \pm q_{+}\right)+a_{2}^{3},
\end{aligned}
$$

where

$$
q_{ \pm}=\sqrt{\left(J-(b-a) \pm \Omega_{R}\right)^{2}+4 \Omega^{2}}
$$

The susceptibility of the system

$$
\chi=\frac{\frac{\mu_{a}^{2}}{\Omega_{R}} \operatorname{sh} \beta \Omega_{R}+\beta e^{-\beta(J-(b-a))}\left(\mu_{a}+\mu_{a b}\right)^{2}}{\operatorname{ch} \beta \Omega_{R}+e^{-\beta(J-(b-a))}}
$$

has the maximum at $\Omega_{R}=0$ and smoothly decreases with the increase of $\Omega_{R}$. Such a behaviour of $\chi\left(\Omega_{R}\right)$ is connected with the dependence of the energy spectrum (5.3) on $\Omega_{R}$ : at $\Omega_{R}=0$ the lowest two branches have a cross-section.

When the electric field is applied the $D$-defect migrates in the direction of the applied field. The dipole moment of the proton subsystem increases with the growth of $N$. Thus the high-energy configurations, such as $D-, L-$ defects and localization of two protons near the same ionic group(with the energy $w$ ) may be realized at values of $E$, which decrease with the bonds number growth. For example, for $N=3, n=4$ at $E_{1}=\frac{J-(b-a)}{2 \mu_{a b}}$ the reconstruction of the spectrum takes place, which reflects the appearance of $D$-defect and the configuration with the energy $w$ on the right end of the chain. 

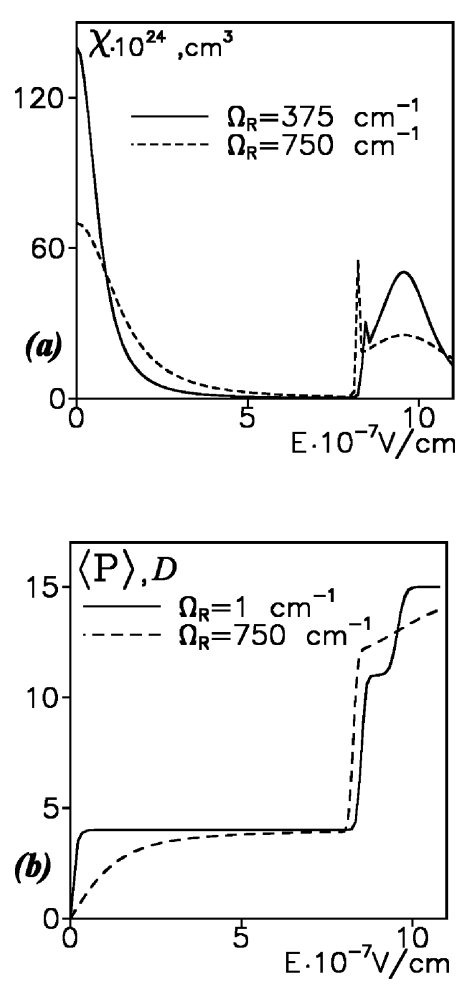

Fig. 10. a) Susceptibility and b) polarization as a functions of $E$ for different $\Omega_{R}, T=100 K, N=3, n=4$.

At $E_{2}=\frac{U+V}{2 \mu_{a}}$ the change of the ground state of the system corresponds to the transfer of all the protons to the right side of the chain, i.e., we have $L$-defect, two $D$-defects and the configuration with the energy $w$. Consequently a sharp increase of $\langle P\rangle$ and maxima of $\chi$ at $E=E_{1}$ and $E=E_{2}$ can be observed in Figs. 10,b,a respectively. For the case of $N=4, n=5$ the dipole moment of the proton subsystem becomes so large, that
$E_{2}>E_{1}$, i.e., at $E_{2}=\frac{J+U+V-(b-a)}{4 \mu_{a}+3 \mu_{a b}}$ the spectrum also is reconstructed and we have two $D$-defects, the short-range proton configuration with the energy $w$ on the right side of the chain and $L$-defect on the left side.

The similarity in the behaviour of proton subsystems can be observed for the cases $N=n$ and $N=n \pm 1$, when the dependence of thermodynamical characteristics of such objects on $E$ is analyzed. The application of external field leads to the pumping of protons in the direction of applied field and, as a result, to the formation of high-energy proton configurations with further increasing of $E$.

\section{ONE PROTON IN THE CHAIN}

As an example of the object with more than one $L$ defects we consider the hydrogen-bonded chain with $N$ bonds and only one proton. The systems with proton motion between hydrogen bond caused by the rotation of a covalent bond connecting a proton to the ionic group should be effective proton wires since it is not necessary for $O H$ groups to be reoriented after every conduction process in a hydrogen-bonded chain, as has been proposed in [17].

The proton motion in a multiminima potential has been studied in [18]. Such objects have anomalous proton polarizability which increases strongly with the increasing of the number of minima.

We have obtained dependences of the energy spectrum on the field strength $E$ using the above mentioned values of the parameters $(b-a), \Omega, \Omega_{0}, \mu_{a}, \mu_{a b}$ and various values of $\Omega_{R}$. For $\Omega=\Omega_{0}=0$ the energy spectrum can be written as:

$$
\begin{aligned}
& \left.\lambda_{1,2}=(N-1) V+(b-a) \pm\left((N-1) \mu_{a}+\frac{N}{2} \mu_{a b}\right)\right)+a_{N}^{1}, \\
& \lambda_{3, \ldots, 2 N}=(N-1) V+a_{N}^{1} \\
& + \begin{cases}\left\{\begin{array}{l} 
\pm\left(\frac{\mu_{a b}}{2}+\mu_{a}\right) E \pm p, \\
\cdots \\
\pm(N-2)\left(\frac{\mu_{a b}}{2}+\mu_{a}\right) E \pm p
\end{array}\right. & , \text { for odd } N \\
\left\{\begin{array}{l} 
\pm p, \\
\pm\left(\mu_{a b}+2 \mu_{a}\right) E \pm p, \\
\cdots \\
\pm\left(\left(\frac{N}{2}-1\right) \mu_{a b}+(N-2) \mu_{a}\right) E \pm p
\end{array}\right. & , \text { for even } N\end{cases}
\end{aligned}
$$

where $p=\sqrt{\mu_{a}^{2} E^{2}+\Omega_{R}^{2}}$. Consequently $\chi\left(\Omega_{R}\right)$ has two regimes for low temperatures:

$$
\chi \stackrel{\beta \rightarrow \infty}{\rightarrow} \begin{cases}\beta\left((N-1) \mu_{a}+\frac{N}{2} \mu_{a, b}\right)^{2} & , \Omega_{R}<-(b-a) \\
\frac{\mu_{a}^{2}}{\Omega_{R}}+\frac{2 \beta}{N-1}\left\{\begin{array}{ll}
s_{1}, & \text { for odd } N \\
s_{2}, & \text { for even } N
\end{array}, \Omega_{R}>-(b-a)\right.\end{cases}
$$




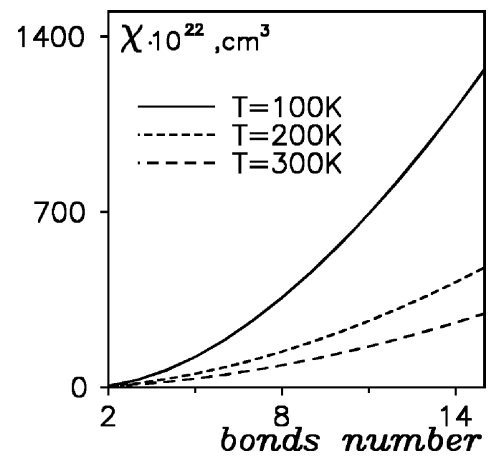

Fig. 11. Static susceptibility of the system with one proton as a function of $N, \Omega=255 \mathrm{~cm}^{-1}, \Omega_{0}=40 \mathrm{~cm}^{-1}$.

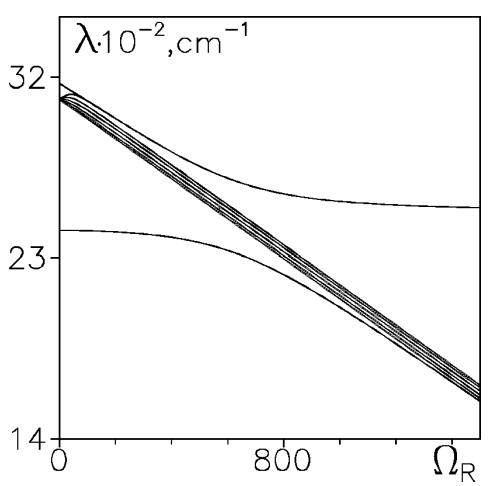

Fig. 12. Dependence of the energy spectrum on $\Omega_{R}, N=8$ (chain with one proton).

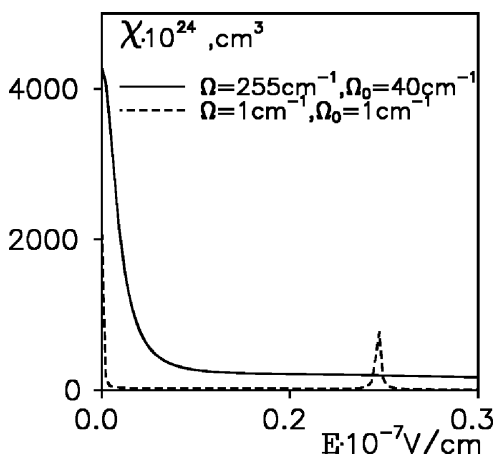

Fig. 13. $\chi(E), N=3, n=1$ for different $\Omega, \Omega_{0}$; $\Omega_{R}=800 \mathrm{~cm}^{-1}, T=50 \mathrm{~K}$.

Here

$$
\begin{aligned}
& s_{1}=\sum_{i=1}^{N / 2}\left((2 i-1)\left(\mu_{a}+\mu_{a, b} / 2\right)\right)^{2}, \\
& s_{2}=\sum_{i=1}^{N / 2-1}\left(i\left(2 \mu_{a}+\mu_{a, b}\right)\right)^{2} .
\end{aligned}
$$

Such dependences are caused by the reconstruction of spectra at $\Omega_{R}=-(b-a)$, i.e., the localization of the proton in one of the inner bonds. We note, that susceptibility of such a model system increases proportionally to $N^{2}$ (see Fig. 11) which corresponds to the results obtained in [18]. It is connected with the increasing of the number of branches with energies close to the excited states for $\Omega_{R}<-(b-a)$ or to the ground state for $\Omega_{R}>-(b-a)$. These $(N-1)$ energy levels with the increasing of $N$ form the energy band (Fig. 12). The tendency to the formation of the band was noted in [13]. For the case of a long but finite H-bonded chain in [19] it was shown that the states of protons both collective (zone) and localized on the ends of the chain occur.

The character of the dependence of an average dipole moment $\langle P\rangle$ on the field $E$ does not change qualitatively with the number of bonds; only the saturation values of the dipole moment (equal to $\left.(N-1) \mu_{a}+\frac{N}{2} \mu_{a b}\right)$ undergo the changes. In the range of $\Omega_{R}>-(b-a)\langle P(E)\rangle$ sharply increases at

$$
E^{*}=\frac{(b-a) \mu_{1}-\sqrt{(b-a)^{2} \mu_{a}^{2}+\Omega_{R}^{2}\left(\mu_{1}^{2}-\mu_{a}^{2}\right)}}{\mu_{1}^{2}-\mu_{a}^{2}},
$$

where $\mu_{1}=\mu_{a}+\mu_{a b}$ for various $N$. The dependence of $\chi(E)$ is almost identical for various $N$ within a wide range of the strength of the electric field (except for a small vicinity of $E=0)$. For example, the function $\chi(E)$ for the chain with $N=3$ hydrogen bonds is presented in Fig. 13. Such a peculiarity in the behaviour of $\langle P\rangle$ and $\chi$ for $\Omega_{R}>-(b-a)$ is related to the moving of delocalized proton from the inner towards the outer hydrogen bond in the direction of the applied field.

\section{CONCLUSIONS}

In comparison with the reduced basis model [12] the proposed approach which includes the proton transfer within the hydrogen bond as well as the orientational hopping between the neighbouring bonds, provides a possibility to describe the proton transport process. Despite the simplicity (one-dimensional system, the description of phonon-assistent hopping as the pseudo-tunneling effect) the presented scheme makes it possible to elucidate the essential influence of orientational motion on the energy spectrum of the proton subsystem of hydrogen bonded chain. As a result of the redistribution of protons in the chain the ground state is changed. The thermodynamical functions of the system (polarization, susceptibility) depend strongly on the external electric field $E$ and on the frequency of orientational pseudotunneling $\Omega_{R}$. The proposed scheme also gives a possibility to describe the dynamics of $L$ - and $D$-defects and their influence on thermodynamical properties of the system.

This model can be considered as the initial point for the description of proton transport, phonon-assistent hopping and calculation of kinetic coefficients for such real physical objects as superprotonic conductors. 


\title{
ACKNOWLEDGMENTS
}

This work was supported by the Ukrainian State Committee for Science and Technology, project No 2.3/806.

[1] G. Raimbault, F. Romain and A. Lautie, J. Raman Spectrosc. 23, 147 (1992).

[2] B. V. Merinov, A. I. Baranov, L. A. Shuvalov, Crystallografiya 35, 355 (1990).

[3] M. Kamoun, M. Halouani, A. Daoud, Phase Transitions 9, 327 (1987).

[4] K. Lukaszewicz, A. Pietraszko, M. A. Augustyniak, Acta Cryst. C49, 430 (1993).

[5] B. Hilczer, A. Pawlowski, Ferroelectrics 104, 383 (1990).

[6] Th. Dippel, N. Hainovsky, K. D. Kreuer, W. Munch, Ferroelectrics 167, 59 (1995).

[7] M. Hubman, Z.Physik B32, 127 (1979).

[8] R. Hassan, E. Campbell, J. Chem. Phys. 97, 4326 (1992).

[9] A. von Hippel, J. Chem. Phys. 54, 145 (1971).

[10] G. Zundel, J. Mol. Struct. 177, 43 (1988).

[11] M. Eckert, G. Zundel, J. Phys. Chem. 92, 7016 (1988).

[12] I. V. Stasyuk, A. L. Ivankiv, Mod. Phys. Lett. B6, 85
(1992).

[13] І. В. Стасюк, О. Л. Іванків, Укр. фіз. журн. 36, 817 (1991).

[14] C. P. Smyth, in Molecular Interactions, edited by H. Ratajczak and W. J. Orville-Thomas (John Wiley and Sons, 1981), Vol.II, p.306.

[15] Here the electric field is the local reaction field at a hydrogen bond in solution or the local crystal field in solid. The local field strength is of the order $10^{7} \mathrm{~V} / \mathrm{cm}[10,14]$.

[16] D. Eisenberg, W. Kauzman, Structure and Properties of Water (in russian, Leningrad, 1975).

[17] В. Я. Антонченко, А. С. Давыдов, В. В. Ильин, Основы физики воды (Наукова Думка, Киев,1991).

[18] G. Zundel, J. Mol. Struct. 181, 141 (1988).

[19] І. В. Стасюк, С. Стаменкович, О. Л. Іванків, Укр. фіз. журн. 36, 1305 (1991).

\section{ОРІЄНТАЦЙНО-ТУНЕЛЬНА МОДЕЛЬ ОДНОВИМІРНИХ МОЛЕКУЛЯРНИХ СИСТЕМ З ВОДНЕВИМИ ЗВ'ЯЗКАМИ}

\author{
I. В. Стасюк, О. Л. Іванків, Н. І. Павленко \\ Інститут фізики конденсованих систем Національної академї̈ наук України \\ Україна, 290011, Лъвів, вул. Свениічъкого, 1
}

Розглянена молекулярна система з $N$ йонних груп, поєднаних між собою водневими зв'язками. Для опису динаміки протонної підсистеми враховано як рух протона в двомінімумному потенціялі на водневому зв'язку з частотою тунелювання $\Omega$ (що супроводжується виникненням т.зв. йонних дефектів), так і орієнтаційні перескоки протона між сусідніми водневими зв'язками з частотою псевдотунелювання $\Omega_{R}$ в результаті ротацій йонних груп (дефекти Б'єррума). Описаний вище механізм дозволяє розглядати протонний перенос вздовж ланцюжка водневих зв'язків.

Досліджено вплив орієнтаційних перескоків на енергетичний спектр скінчених комплексів з водневими зв'язками; виявлено можливість перебудови основного стану системи та перерозподілу протонів в ланцюжку при цьому. Показано, що характер поведінки термодинамічних функцій (дипольного моменту і діелектричної сприйнятливості) істотно залежить від частоти орієнтаційного псевдотунелювання $\Omega_{R}$, а також від напруженості зовнішнього електричного поля.

В рамках запропонованої моделі описано системи з $L$ - (кількість протонів $n=N-1)$ і $D(n=N+1)$ дефектами. Як приклад об'єкта з більш ніж одним $L$-дефектом розглядається модельна задача про рух одного протона в ланцюжку з $N$ водневими зв'язками. Досліджено динаміку $L$ - і $D$-дефектів, а також проаналізовано їх вплив на термодинамічні властивості протонної підсистеми.

Запропонована модель може розглядатись як вихідна для опису протонного транспорту та обчислення кінетичних коефіцієнтів в реальних фізичних об’єктах, зокрема таких, як суперйонні кристали. 